\title{
Low dose allopurinol is associated with modest but significant improvements in glycemic control (GC) and glycemic variability (GV) in patients with type 2 diabetes mellitus: A cross-sectional study
}

\author{
Manal M. Alem ( $\sim$ malem@iau.edu.sa ) \\ Imam Abdulrahman Bin Faisal University
}

\section{Research Article}

Keywords: Type 2 Diabetes Mellitus, glycemic control, glycated hemoglobin, glycemic variability, allopurinol, xanthine oxidase

Posted Date: May 12th, 2021

DOI: https://doi.org/10.21203/rs.3.rs-491373/v1

License: (c) (1) This work is licensed under a Creative Commons Attribution 4.0 International License. Read Full License 


\section{Abstract}

\section{Background}

Type 2 diabetes mellitus (DM), gout, and asymptomatic hyperuricemia are inter-connected pathologies. Glycemic control (GC), involving a range of treatments is central to the management of DM, whereas allopurinol continues to be the most widely recommended urate lowering agent. Allopurinol has been shown to possess anti-oxidant properties: this study explores the favorable potential effect of allopurinol on glucose homeostasis.

\section{Methods}

This is an observational study with a cross-sectional design performed on patients with type 2 diabetes mellitus (DM), recruited from centers in Saudi Arabia. Patients were divided into two groups; allopurinol users; (for gout or asymptomatic hyperuricemia) and matching control patients. Patient demographics, co-morbid conditions, biochemical tests, and pharmacological treatments were extracted from electronic records to investigate the effect of allopurinol therapy on Glycemic control (GC), as assessed by glycated haemoglobin (HbA1c as primary endpoint), and on parameters of glycaemic variability (GV) (secondary endpoints).

\section{Results}

A total of 194 patients with type $2 \mathrm{DM}$ were recruited (97 in both groups). The two groups were matched for age and sex: mean age: 59.4 years, $73 \%$ males in the allopurinol group vs 59.6 years, $73 \%$ males in the control group. Allopurinol, daily dose $100 \mathrm{mg}$, was prescribed for $77 \%$ of the patients, with median duration of 39.5 months treatment. $\mathrm{HbA} 1 \mathrm{c}$ values were; $6.90 \%(6.20,7.80)$ in the allopurinol group vs $7.30 \%(6.60,8.40)$ in the control group $(P=0.010)$. Parameters of GV were calculated from 3 consecutive fasting blood sugar (FBS) readings: variability independent of the mean (VIM) was 0.140 in the allopurinol group vs 0.987 in the control group $(P<0.001)$.

\section{Conclusion}

Concomitant low-dose allopurinol therapy in patients with type $2 \mathrm{DM}$ was associated with modest but significant improvements in GC and GV.

\section{Introduction}

Type 2 diabetes mellitus (DM) has a global prevalence of 9.3\% (2019), but it is expected to rise to $10.2 \%$ by 2030 , and $10.9 \%$ by 2045 (1). Such alarming numbers with the well-recognized macro- and microvascular complications constitute serious threats to the quality of life/ life expectancy of affected patients. The UK Prospective Diabetes Study (UKPDS) has shown that glycemic control (GC) is the main therapeutic strategy to lower the risk of microvascular complications in newly diagnosed patients with type 2 diabetes(2). A post-trial monitoring (10 years) has extended the benefits to include macrovascular 
complications. In summary, intensive therapy (in the sulfonylurea-insulin group) reduced the relative risk of microvascular disease by $24 \%$, myocardial infarction by $15 \%$ and death from any cause by $13 \%$. Correspondingly, in the metformin group, the relative risk reductions were $33 \%$ for myocardial infarction and $27 \%$ for death from any cause (3). To achieve GC, detailed pharmacologic approaches have been described by the American Diabetes Association (ADA), issued as part of: Standards of Medical Care in Diabetes 2021(4;5).

Glycemic variability (GV) is a more recently identified phenomenon describing fluctuations or oscillations in blood glucose concentrations which can adversely affect outcomes (6). GV can be classified as shortterm GV (referring to within- or between days fluctuations) and long-term GV (referring to fluctuations over several weeks or months)(7). These phenomena, independent of glycemic control, have been shown to be additional risks for diabetes-related complications; micro- and macro-vascular, and mortality (7). In a post-hoc cohort analysis of data from the Antihypertensive and Lipid-Lowering treatment to prevent Heart Attack Trial (ALLHAT) that included patients with and without diabetes, it was shown that higher GV was associated with increased risk of all-cause mortality (8). Similar findings have been reported by other studies confirming the association between GV and diabetic micro-, macro-vascular complications, and all-cause mortality 7;9;10).

The pathogenesis of diabetes, and its complications is multi-factorial and involves complex pathways including the inter-connection between diabetes, free radicals, endothelial function and diabetes-related complications (11). Hyperglycemia has been shown to contribute to free radical generation, oxidative stress, endothelial dysfunction and, finally diabetes-related complications (12). This series of events was discovered more than 30 years ago which introduced the concept that agents with antioxidant properties should be part of the therapeutic strategies in diabetes care(13).

One of the key enzyme systems involved in oxidative stress is xanthine oxidoreductase (XOR). This enzyme system exists in two forms; xanthine dehydrogenase (XDH) and xanthine oxidase (XO)(14). The latter is a source of reactive oxygen species that contributes to oxidative stress in the diabetic state, where its activity was found to be significantly higher than in control subjects (15), and correlating positively with biomarkers of glycemic control (glycated hemoglobin, HBA1c)(15). Such positive and significant correlation between $\mathrm{XO}$ activity and glycemic control has also been established in patients with multiple cardiovascular risk factors $(16)$, and also in the general population $(17 ; 18)$. Further smallscale research studies have showed the benefits of allopurinol ( $\leq 300 \mathrm{mg} / \mathrm{day}$ ) on GC, as expressed via the homeostatic model assessment of insulin resistance (HOMA-IR), in patients with asymptomatic hyperuricemia over treatment periods of 3 months (19), and 3 years (20), respectively.

This present observational study sought to extend these findings beyond GC to GV: to explore whether or not concomitant allopurinol therapy (as a xanthine oxidase inhibitor prescribed for gout or asymptomatic hyperuricemia) might have a beneficial effect on GC or GV in our local population with type 2 DM.

\section{Methods}




\section{Study Design and patients identification}

This analytical observation study was designed to study the association of concomitant allopurinol therapy on GC in patients with type $2 \mathrm{DM}$ : $\mathrm{HbA} 1 \mathrm{c}$ was the primary endpoint. The secondary endpoint was the corresponding association with GV.

The conduct and reporting of the study followed the recommended guidelines of the STROBE Statement: The Strengthening the Reporting of Observational Studies in Epidemiology(21). Eligible patients were identified from the diabetic centre and the general medical clinics of King Fahd Hospital of the University (KFHU), Al Khobar. In addition, patients were also recruited from the family and community medicine centre of Imam Abdulrahman bin Faisal University campus, Dammam. Patient search and identification were conducted by hospital pharmacy staff via access to the computerized filing system in KFHU, with straight numeric identification of each patient, primarily according to their prescribed medication (Figure 1). After identifying patients for the allopurinol group, the control patients were identified by one-to-one matching based on date of birth (year), and sex.

\section{Ethical Approval and consent process}

The study protocol was approved by the Institutional Review Board (IRB-2020-05-247), Deanship of Scientific Research, Imam Abdulrahman Bin Faisal University, Dammam, Saudi Arabia. Since the study does not involve any intervention, a waiver of informed consent from the participants was obtained. However, where data were incomplete (particularly in relation to the duration of allopurinol treatment), a protocol amendment was approved whereby informed verbal consent (via telephone conversation) was obtained for patients to provide such missing information. This consent process/protocol amendment was approved by the Institutional Review Board. The study was conducted in accordance with the Declaration of Helsinki, the ICH Harmonized Tripartite Good Clinical Practice Guidelines, and the laws of Saudi Arabia.

\section{Inclusion and Exclusion Criteria}

\section{Inclusion criteria}

- Age $>18$ years

- Type 2 DM diagnosed using American Diabetes Association criteria and on pharmacological treatment (search terms- Figure 1)

- Concomitant allopurinol therapy (with any dose) for the active arm only

\section{Exclusion criteria}

- Type 1 DM

- Active (known) malignant disease 


\section{Variables collected}

The following patient demographic information was collected: reported co-morbid conditions, DM medications, and other medications, outpatient reports and, where appropriate, details of allopurinol treatment (indication, start date and dose), presence/absence of complications e.g. renal stone disease. Biochemical variables were also collected, including $\mathrm{HbA} 1 \mathrm{c}$ and matching 3 fasting blood sugar readings: from these measurements, GC and GV were calculated.

\section{Statistical Analysis}

Baseline data are reported as mean \pm Standard Deviation (SD) for continuous variables; number and percentages for categorical variables. Median and interquartile ranges were used for non-normally distributed data. Comparison of the two patients groups was by two-sample T-test and confidence intervals (Cl): the Mann-Whitney test and chi-square test where appropriate. Correlation analyses were performed using Spearman correlation coefficients (rho). The primary endpoint was the association of allopurinol use with $\mathrm{HbA1c}$. Secondary endpoint was the association of allopurinol therapy with GV. All statistical analyses were performed using Minitab statistical software (version18, Minitab Inc., State College, PA, USA). A value of $\mathrm{p} \otimes 0.05$ was considered statistically significant.

\section{Measures of glycemic variability}

Availability of FBS readings, matched to the dates for HbA1c measurements, was available for 94 patients in the allopurinol group and 96 patients in the control group. Sufficient data were available to allow the calculation of dispersion or variability in 78 patients in the allopurinol group and 79 patients in the control group. Using the average of FBS readings, the calculation of visit-to-visit variability (VVV) of FBS was defined by more than one statistic. The first is the intra-individual SD across the visits, the second is the coefficient of variation (defined as the ratio of the SD/ the mean FBS $\times 100$ ). These two statistics are correlated with the mean, tending to increase as the mean increases. A more appropriate statistic is the variability independent of the mean (VIM), that is calculated as $S D \times 100 / \mathrm{Mean}^{\beta}$, where $\beta$ is the regression coefficient based on natural logarithm of SD on natural logarithm of Mean FBS, and compared using Welch T-test(8).

\section{Sample size}

Using our previous results (22), and assuming a pooled standard deviation of 2.05 units, the study would require a sample size of 89 patients in each group (a total sample size of 178 patients) to achieve a $90 \%$ power and a level of significance of $5 \%$ (two-sided), to detect a true difference of $1 \%$ in glycated hemoglobin means between allopurinol users, and control subjects.

\section{Results}


The search process enabled the inclusion of 194 patients into the study, and 97 patients were in each group. Patients in the allopurinol group were matched to control group for age and sex, and for mean achieved blood pressure and heart rate: respectively, 133/79, 80 vs 132/79 $\mathrm{mmHg}, 80$ beats/min). The allopurinol group had significantly higher body weight and body mass index (BMI) (estimated differences of, respectively, $7 \mathrm{Kg}(\mathrm{Cl} ; 1,13, \mathrm{P}=0.020)$ and $2.1 \mathrm{Kg} / \mathrm{m}^{2}(\mathrm{Cl} ; 0.2,4, \mathrm{P}=0.029)$. In terms of the co-morbid conditions, the two groups were well matched (see Table 1 ) apart from the prevalence of a history of systemic hypertension and chronic kidney disease which were significantly higher in allopurinol group; respectively, $85.6 \%$ vs $67 \%(P=0.002)$ and $36.1 \%$ vs $7.2 \%(P<0.001)$ (Table 1$)$. Further classification of the patients based on estimated GFR (calculated by the abbreviated MDRD equation:

$186 \times(\text { Creatinine/88.4 })^{-1.154} \times(\text { Age })^{-0.203} \times(0.742$ if female $) \times(1.210$ if black $)$.

In general, across the stages, renal impairment was more prevalent in the allopurinol group. This included 12 patients in the allopurinol group (vs none in the control group) who were maintained on renal replacement therapy: 2 on hemodialysis and 10 on peritoneal dialysis.

\section{Allopurinol indications and dose}

Allopurinol therapy with indication, dose, and duration (in months before $\mathrm{HbA} 1 \mathrm{c}$ readings) are listed in table 1.The most frequent dose was $100 \mathrm{mg} /$ day in $77.3 \%$ of the whole group, while the median duration for allopurinol therapy was 39.5 months $(10.95,82.78)$

\section{Comparisons of parameters of glycemic control (GC)}

$\mathrm{HbA1c}$ readings were compared between the two groups and showed a small yet significant difference in favor of allopurinol therapy. The estimated difference was $-0.5 \%,(\mathrm{Cl}:-0.8,-0.1)(P=0.010)($ Table 1$)$.

\section{Sensitivity analysis}

Advanced chronic kidney disease (CKD) is associated with shortened red blood cells lifespan which might reduce $\mathrm{HbA} 1 \mathrm{c}$ readings. Accordingly, the $\mathrm{HbA} 1 \mathrm{ccomparison}$ was repeated after removal of the 12 patients on renal replacement therapy (85 allopurinol patients' vs 97 control patients). The estimated difference obtained for $\mathrm{HbA} 1 \mathrm{c}$ was $-0.4 \%$, $(\mathrm{Cl}:-0.7,-0.0)(P=0.033)$.

\section{Comparisons of parameters of glycemic variability (GV)}

- Comparing FBS readings between the two groups is demonstrated in (Table 1) as medians with interquartile ranges $(\mathrm{Q} 1, \mathrm{Q} 3)$ with a trend towards lower reading in favor of allopurinol users, with an estimated difference of $-8 \mathrm{mg} / \mathrm{dL}(\mathrm{Cl}:-17.33,1)(P=0.072)$.

- Comparing the range of FBS readings with the minimum and maximum values were; 84.33 , and $287.00 \mathrm{mg} / \mathrm{dL}$ in the allopurinol group, as compared with 57.00 , and $372.00 \mathrm{mg} / \mathrm{dL}$ in the control group (See Fig. 2). 
- Comparisons of standard deviation, and coefficient of variation showed a trend for less dispersion in FBS readings in allopurinol users (Table 1 ).

- Comparisons of the VIM showed a statistically significant results in favor of allopurinol therapy $(P<0.001)$ (Table 1 and Figure 3 ).

\section{Correlation between GC and GV indices}

By studying the correlation between two parameters of glycemic control; $\mathrm{HbA1c}$, and FBS, with an index of GV; VIM, correlation analysis revealed that FBS was not correlated with VIM in both groups; Spearman correlation coefficients (rho); $(r=-0.018, P=0.876)$ in allopurinol group, and $(r=0.027, P=0.816)$ in control group. While HbA1c had no correlation with VIM in allopurinol group ( $r=0.044, P=0.701)$, it had a significant positive correlation in control group $(r=0.273, P=0.015)$.

\section{Comparisons of other biochemical parameters}

As expected, the renal biochemical parameters (blood urea nitrogen, serum creatinine and serum uric acid concentrations) were higher in the allopurinol group (Table 1). There were no clinically relevant differences for sodium, potassium or chloride.

\section{Comparisons of pharmacological therapy}

The two groups were matched for diabetes mellitus medications but they differed slightly in the use of angiotensin receptor blockers (52.6\% in the allopurinol group vs $36 \%$ in the control group), beta blockers and calcium channel blockers ( $49.5 \%$ vs $27.8 \%)$. There also were small differences for diuretic and aspirin use (Table 2).

\section{Discussion}

In this observational study, it has been shown that low dose allopurinol therapy in patients with type 2 diabetes mellitus was associated with modest, yet significant effect on GC, as assessed by the most widely used biochemical marker, HbA1c. In addition, such therapy was also associated with a significant effect on GV, as assessed by a parameter statistically independent from the mean FBS values (variability independent from the mean). The following are the immediate and obvious questions:

- Is the magnitude of the improvement in GC of clinical significance?

- Is the magnitude of the improvement in GV of clinical significance?

- Do these two parameters/phenomena correlate?

Improvement in GC: what is a clinically significant drop in HbA1c?

The UKPDS has shown that $1 \%$ reduction in $\mathrm{HbA} 1 \mathrm{c}$ was associated with risk reduction of $21 \%$ for any endpoint related to diabetes; $21 \%$ for death related to diabetes; $14 \%$ for myocardial infarction and $37 \%$ for microvascular complications (23). The study emphasized also that, due to the lack of thresholds of 
glycemia for those complications, any reduction in $\mathrm{HbA} 1 \mathrm{c}$ would be likely to reduce the risk of these complications, especially if the concentration approached the normal range (23). Comparisons of the reduction achieved in this study $(-0.5 \%$ with allopurinol) with the effects of established antidiabetic drug groups in patients with type $2 \mathrm{DM}$ but with sub-optimal control are as follows. Glucagon-like peptide 1(GLP-1) agonists (e.g. exenatide, liraglutide, and dulaglutide) have been shown to achieve -0.55 to -1.38 $\%$ reduction in $\mathrm{HbA} 1 \mathrm{c}(24 ; 25)$; dipeptidyl peptidase 4 (DDP 4) inhibitors (e.g. vildagliptin and sitagliptin) can achieve -0.73 , and $-0.74 \%$ reduction, respectively(26);sodium-glucose co-transporter 2 (SGLT 2) inhibitors (e.g. dapagliflozin) can achieve -0.50 to $0.66 \%$ reduction (27-29), while insulin glargine can achieve -0.9 to $-1.11 \%$ reduction( $30 ; 31)$. Accordingly, the reduction achieved by low-dose allopurinol therapy in this study appears to be clinically significant.

A further consideration is that patients in the allopurinol group had higher percentage of use of ARBs, CCBs, beta-blockers, and diuretics. For example, beta blockers and diuretics are known to have negative effects on glucose homeostasis(32), raising the possibility of an otherwise greater beneficial effect from allopurinol. In that regard, the magnitude of drop inHbA1c in this study was smaller than was observed in another sample of our population in a previous study of similar sample size, allopurinol dose and duration $(-0.8 \%$ reduction).

Regarding, GV: there is lack of agreement in the literature on the parameter that best reflects GV; there also is a lack of agreement on how to derive the relevant parameters. For example, one recent study assessed the prognostic significance of GV calculated from HbA1c vs that calculated from FBS and found that GV parameters calculated from FBS readings were more consistent with the metabolic outcomes and complications (10). There also are statistical considerations and methodological considerations (33). However, in an attempt to relate the results obtained in this study to a reference method, the ALLHAT study suggested that VIM is possibly the most robust measure of GV insofar as each unit change in VIM was associated with a higher risk of death; HR $1.014(95 \% \mathrm{Cl} 1.006-1.022)(\mathrm{P}=$ $0.001)(8)$.

With regard to the relationship between GC and GV, the findings of this study identify no direct or definite relationship. This is in agreement with those few studies in the literature that have assessed both phenomena simultaneously using mean amplitude of glycemic excursions (MAGE), calculated over 4872 hours over continuous sub-cutaneous interstitial glucose monitoring. Monnieret al. demonstrated that markers of oxidative stress were higher in type $2 \mathrm{DM}$ patients in comparison with control subjects, and they correlated strongly with GV estimated by MAGE, and not with GC estimated by HbA1c(34). Another two studies by Rizzo et al. and by Kimet al. compared vildagliptin; a DDP 4 inhibitors vs sitagliptin in the first, and vs pioglitazone in the second. Both studies showed that the two arms had a similar effect on GC (HbA1c), however, only vildagliptin had an extra advantage on GV assessed by (MAGE)(35;36). Another important finding was that GV reduction was associated with reduction of markers of oxidative stress and systemic inflammation, an association that was not found to exist with GC indices (35).According to the current evidence, and the correlation analysis in this study, both GC and GV can co-exist in patients 
but it is likely that different pathways are involved and differential responses to pharmacological intervention.

The inter-relationships between glucose homeostasis and urate metabolism. So-called asymptomatic hyperuricemia, as a persistent biochemical abnormality, not only predisposes to gout but is also an independent risk factor for type 2 DM $(37 ; 38)$. Allopurinol, as an established xanthine oxidase inhibitor which continues as the first-line urate lowering drug even in CKD patients (stage $\geq 3$ ), has been shown to have benefits in type $2 \mathrm{DM}$ patients $(38,39)$. A recent large population-based cohort study showed that elderly patients with diabetes mellitus who were prescribed allopurinol and followed for over 4 years had reduced mortality and cardiovascular outcomes (39). Perhaps these observations can be attributed to a favorable metabolic profile "at least partially": this might encourage health care professionals to lower the threshold for the prescription of allopurinol.

\section{Limitations}

This study has a number of limitations. Firstly, the observational design does not allow cause and effect relationship to be established. There also were differences between the groups in relation to renal function: assessing glycemic control by glycated hemoglobin may be less reliable in patients with advanced CKD. Other glycated proteins "that are still under research"(40) are not routinely available in our hospitals. The concept of glycemic variability is still new in the literature; there is still no agreement/consensus on the best index of GV.

\section{Conclusion}

In this observational study, it has been shown that low dose allopurinol therapy in patients with type 2 DM was associated with a favorable impact on glycemic control. Although the benefit was of a lesser magnitude, it is expected to be of a clinical significance since it is comparable to the effects of antidiabetic medications. In addition, allopurinol use was associated with an additional favorable effect on GV, a new risk factor that has been shown to be independently associated with DM complications. Allopurinol is an old drug with an established safety record, no association with hypoglycemia and with low cost. Accordingly, future research is warranted to determine whether or not allopurinol has beneficial effects on not only on glucose homeostasis but also on the micro- and macro-vascular complications in patients with type $2 \mathrm{DM}$.

\section{List Of Abbreviations}

(DM) diabetes mellitus

(GC), glycemic control

(GV) glycaemic variability 
(UKPDS)UK Prospective Diabetes Study

(ADA),American Diabetes Association

(ALLHAT)Antihypertensive and Lipid-Lowering treatment to prevent Heart Attack Trial

(XOR) xanthine oxidoreductase

$(\mathrm{XDH})$ xanthine dehydrogenase

(XO) xanthine oxidase

(STROBE)Strengthening the Reporting of Observational Studies in Epidemiology

(SD)Standard Deviation

(CKD) chronic kidney disease

(Cl) confidence intervals

(VVV) visit-to-visit variability

(VIM) variability independent of the mean

(FBS) fasting blood sugar

(HbA1c) glycated hemoglobin

(GLP-1) glucagon-like peptide 1

(DDP 4) dipeptidyl peptidase 4

(SGLT 2) sodium-glucose co-transporter 2

(MAGE) mean amplitude of glycemic excursions

\section{Declarations}

\section{Ethics approval and consent to participate}

The study protocol was approved by the Institutional Review Board (IRB-2020-05-247), Deanship of Scientific Research, Imam Abdulrahman Bin Faisal University, Dammam, Saudi Arabia. Since the study does not involve any intervention, a waiver of informed consent from the participants was obtained. However, where data were incomplete (particularly in relation to the duration of allopurinol treatment), a protocol amendment was approved whereby informed verbal consent (via telephone conversation) was obtained for patients to provide such missing information. This consent process/protocol amendment 
was approved by the Institutional Review Board. The study was conducted in accordance with the Declaration of Helsinki, the ICH Harmonized Tripartite Good Clinical Practice Guidelines, and the laws of Saudi Arabia.

\section{Consent for publication}

The author grants BMC Pharmacology and Toxicology the license of publishing this study

\section{Availability of data and materials}

The data that support the findings of this study are available on request from the corresponding author (MM. Alem).

\section{Competing interests}

The author declares no conflict of interest.

\section{Funding}

This research has not been funded by any source

\section{Authors' contributions}

A single author designed, executed this study, and prepared this manuscript.

\section{Acknowledgments}

The author would like to thank the pharmacy staff in King Fahd Hospital of the University (KFHU), Al Khobar.

\section{References}

1. Saeedi P, Petersohn I, Salpea P, Malanda B, Karuranga S, Unwin N, et al. Global and regional diabetes prevalence estimates for 2019 and projections for 2030 and 2045: Results from the International Diabetes Federation Diabetes Atlas, 9(th) edition. Diabetes Res Clin Pract 2019 Nov;157:107843.

2. Intensive blood-glucose control with sulphonylureas or insulin compared with conventional treatment and risk of complications in patients with type 2 diabetes (UKPDS 33). UK Prospective Diabetes Study (UKPDS) Group. Lancet 1998 Sep 12;352(9131):837 - 53.

3. Holman RR, Paul SK, Bethel MA, Matthews DR, Neil HA. 10-year follow-up of intensive glucose control in type 2 diabetes. N Engl J Med 2008 Oct 9;359(15):1577-89.

4. 9. Pharmacologic Approaches to Glycemic Treatment: Standards of Medical Care in Diabetes-2021. Diabetes Care 2021 Jan;44(Suppl 1):S111-S124. 
5. 6. Glycemic Targets: Standards of Medical Care in Diabetes-2021. Diabetes Care 2021 Jan;44(Suppl 1):S73-S84.

6. Ceriello A, Esposito K, Piconi L, Ihnat MA, Thorpe JE, Testa R, et al. Oscillating glucose is more deleterious to endothelial function and oxidative stress than mean glucose in normal and type 2 diabetic patients. Diabetes 2008 May;57(5):1349-54.

7. Gorst C, Kwok CS, Aslam S, Buchan I, Kontopantelis E, Myint PK, et al. Long-term Glycemic Variability and Risk of Adverse Outcomes: A Systematic Review and Meta-analysis. Diabetes Care 2015 Dec;38(12):2354-69.

8. Echouffo-Tcheugui JB, Zhao S, Brock G, Matsouaka RA, Kline D, Joseph JJ. Visit-to-Visit Glycemic Variability and Risks of Cardiovascular Events and All-Cause Mortality: The ALLHAT Study. Diabetes Care 2019 Mar;42(3):486-93.

9. Zinman B, Marso SP, Poulter NR, Emerson SS, Pieber TR, Pratley RE, et al. Day-to-day fasting glycaemic variability in DEVOTE: associations with severe hypoglycaemia and cardiovascular outcomes (DEVOTE 2). Diabetologia 2018 Jan;61(1):48-57.

10. Slieker RC, van der Heijden AAWH, Nijpels G, Elders PJM, 't Hart LM, Beulens JWJ. Visit-to-visit variability of glycemia and vascular complications: the Hoorn Diabetes Care System cohort. Cardiovasc Diabetol 2019 Dec 12;18(1):170.

11. Maritim AC, Sanders RA, Watkins JB, III. Diabetes, oxidative stress, and antioxidants: a review. J Biochem Mol Toxicol 2003;17(1):24-38.

12. Ceriello A. Oxidative stress and diabetes-associated complications. Endocr Pract 2006 Jan;12 Suppl $1: 60-2$.

13. Hunt JV, Dean RT, Wolff SP. Hydroxyl radical production and autoxidative glycosylation. Glucose autoxidation as the cause of protein damage in the experimental glycation model of diabetes mellitus and ageing. Biochem J 1988 Nov 15;256(1):205-12.

14. Nishino T, Okamoto $\mathrm{K}$. Mechanistic insights into xanthine oxidoreductase from development studies of candidate drugs to treat hyperuricemia and gout. J Biol Inorg Chem 2015 Mar;20(2):195-207.

15. Kuppusamy UR, Indran M, Rokiah P. Glycaemic control in relation to xanthine oxidase and antioxidant indices in Malaysian Type 2 diabetes patients. Diabet Med 2005 Oct;22(10):1343-6.

16. Fujimura Y, Yamauchi Y, Murase T, Nakamura T, Fujita SI, Fujisaka T, et al. Relationship between plasma xanthine oxidoreductase activity and left ventricular ejection fraction and hypertrophy among cardiac patients. PLoS One 2017;12(8):e0182699.

17. Furuhashi M, Matsumoto M, Tanaka M, Moniwa N, Murase T, Nakamura T, et al. Plasma Xanthine Oxidoreductase Activity as a Novel Biomarker of Metabolic Disorders in a General Population. Circ J 2018 Jun 25;82(7):1892-9.

18. Washio KW, Kusunoki Y, Murase T, Nakamura T, Osugi K, Ohigashi M, et al. Xanthine oxidoreductase activity is correlated with insulin resistance and subclinical inflammation in young humans. Metabolism 2017 May;70:51-6. 
19. Takir M, Kostek O, Ozkok A, Elcioglu OC, Bakan A, Erek A, et al. Lowering Uric Acid With Allopurinol Improves Insulin Resistance and Systemic Inflammation in Asymptomatic Hyperuricemia. J Investig Med 2015 Dec;63(8):924-9.

20. Liu P, Wang H, Zhang F, Chen Y, Wang D, Wang Y. The Effects of Allopurinol on the Carotid Intimamedia Thickness in Patients with Type 2 Diabetes and Asymptomatic Hyperuricemia: A Three-year Randomized Parallel-controlled Study. Intern Med 2015;54(17):2129-37.

21. von EE, Altman DG, Egger M, Pocock SJ, Gotzsche PC, Vandenbroucke JP. The Strengthening the Reporting of Observational Studies in Epidemiology (STROBE) statement: guidelines for reporting observational studies. PLoS Med 2007 Oct 16;4(10):e296.

22. Alem MM, Aldosari SR, Alkahmous AA, Obad AS, Fagir NM, Al-Ghamdi BS. Effect of Long-Term Allopurinol Therapy on Left Ventricular Mass Index in Patients with Ischemic Heart Disease; A CrossSectional Study. Vasc Health Risk Manag 2019;15:539-50.

23. Stratton IM, Adler Al, Neil HA, Matthews DR, Manley SE, Cull CA, et al. Association of glycaemia with macrovascular and microvascular complications of type 2 diabetes (UKPDS 35): prospective observational study. BMJ 2000 Aug 12;321(7258):405 - 12.

24. Htike ZZ, Zaccardi F, Papamargaritis D, Webb DR, Khunti K, Davies MJ. Efficacy and safety of glucagon-like peptide-1 receptor agonists in type 2 diabetes: A systematic review and mixedtreatment comparison analysis. Diabetes Obes Metab 2017 Apr;19(4):524-36.

25. Andreadis P, Karagiannis T, Malandris K, Avgerinos I, Liakos A, Manolopoulos A, et al. Semaglutide for type 2 diabetes mellitus: A systematic review and meta-analysis. Diabetes Obes Metab 2018 Sep;20(9):2255-63.

26. Amori RE, Lau J, Pittas AG. Efficacy and safety of incretin therapy in type 2 diabetes: systematic review and meta-analysis. JAMA 2007 Jul 11;298(2):194-206.

27. Liu XY, Zhang N, Chen R, Zhao JG, Yu P. Efficacy and safety of sodium-glucose cotransporter 2 inhibitors in type 2 diabetes: a meta-analysis of randomized controlled trials for 1 to 2 years. J Diabetes Complications 2015 Nov;29(8):1295-303.

28. Musso G, Gambino R, Cassader M, Pagano G. A novel approach to control hyperglycemia in type 2 diabetes: sodium glucose co-transport (SGLT) inhibitors: systematic review and meta-analysis of randomized trials. Ann Med 2012 Jun;44(4):375-93.

29. Vasilakou D, Karagiannis T, Athanasiadou E, Mainou M, Liakos A, Bekiari E, et al. Sodium-glucose cotransporter 2 inhibitors for type 2 diabetes: a systematic review and meta-analysis. Ann Intern Med 2013 Aug 20;159(4):262-74.

30. Marso SP, McGuire DK, Zinman B, Poulter NR, Emerson SS, Pieber TR, et al. Efficacy and Safety of Degludec versus Glargine in Type 2 Diabetes. N Engl J Med 2017 Aug 24;377(8):723 - 32.

31. Heine RJ, Van Gaal LF, Johns D, Mihm MJ, Widel MH, Brodows RG. Exenatide versus insulin glargine in patients with suboptimally controlled type 2 diabetes: a randomized trial. Ann Intern Med 2005 Oct 18;143(8):559- 69. 
32. Rizos CV, Elisaf MS. Antihypertensive drugs and glucose metabolism. World J Cardiol 2014 Jul 26;6(7):517-30.

33. Lee S, Lee H, Kim Y, Kim E. Effect of DPP-IV Inhibitors on Glycemic Variability in Patients with T2DM: A Systematic Review and Meta-Analysis. Sci Rep 2019 Sep 16;9(1):13296.

34. Monnier L, Mas E, Ginet C, Michel F, Villon L, Cristol JP, et al. Activation of oxidative stress by acute glucose fluctuations compared with sustained chronic hyperglycemia in patients with type 2 diabetes. JAMA 2006 Apr 12;295(14):1681-7.

35. Rizzo MR, Barbieri M, Marfella R, Paolisso G. Reduction of oxidative stress and inflammation by blunting daily acute glucose fluctuations in patients with type 2 diabetes: role of dipeptidyl peptidase-IV inhibition. Diabetes Care 2012 Oct;35(10):2076-82.

36. Kim NH, Kim DL, Kim KJ, Kim NH, Choi KM, Baik SH, et al. Effects of Vildagliptin or Pioglitazone on Glycemic Variability and Oxidative Stress in Patients with Type 2 Diabetes Inadequately Controlled with Metformin Monotherapy: A 16-Week, Randomised, Open Label, Pilot Study. Endocrinol Metab (Seoul) 2017 Jun;32(2):241-7.

37. Bhole V, Choi JW, Kim SW, de VM, Choi H. Serum uric acid levels and the risk of type 2 diabetes: a prospective study. Am J Med 2010 Oct;123(10):957-61.

38. Dehghan A, van HM, Sijbrands EJ, Hofman A, Witteman JC. High serum uric acid as a novel risk factor for type 2 diabetes. Diabetes Care 2008 Feb;31(2):361-2.

39. Weisman A, Tomlinson GA, Lipscombe LL, Perkins BA, Hawker GA. Association between allopurinol and cardiovascular outcomes and all-cause mortality in diabetes: A retrospective, population-based cohort study. Diabetes Obes Metab 2019 Jun;21(6):1322-9.

40. Welsh KJ, Kirkman MS, Sacks DB. Role of Glycated Proteins in the Diagnosis and Management of Diabetes: Research Gaps and Future Directions. Diabetes Care 2016 Aug;39(8):1299-306.

\section{Tables}

Due to technical limitations, the tables are only available as a download in the supplemental files section.

\section{Figures}


Electronic search for suitable patients in King Fahd Hospital of the university and The family and community Medicine Center using the electronic filing system with search terms "Metformin", and/or, "Allopurinol", from Jan 1", 2019 till “June 30"2020
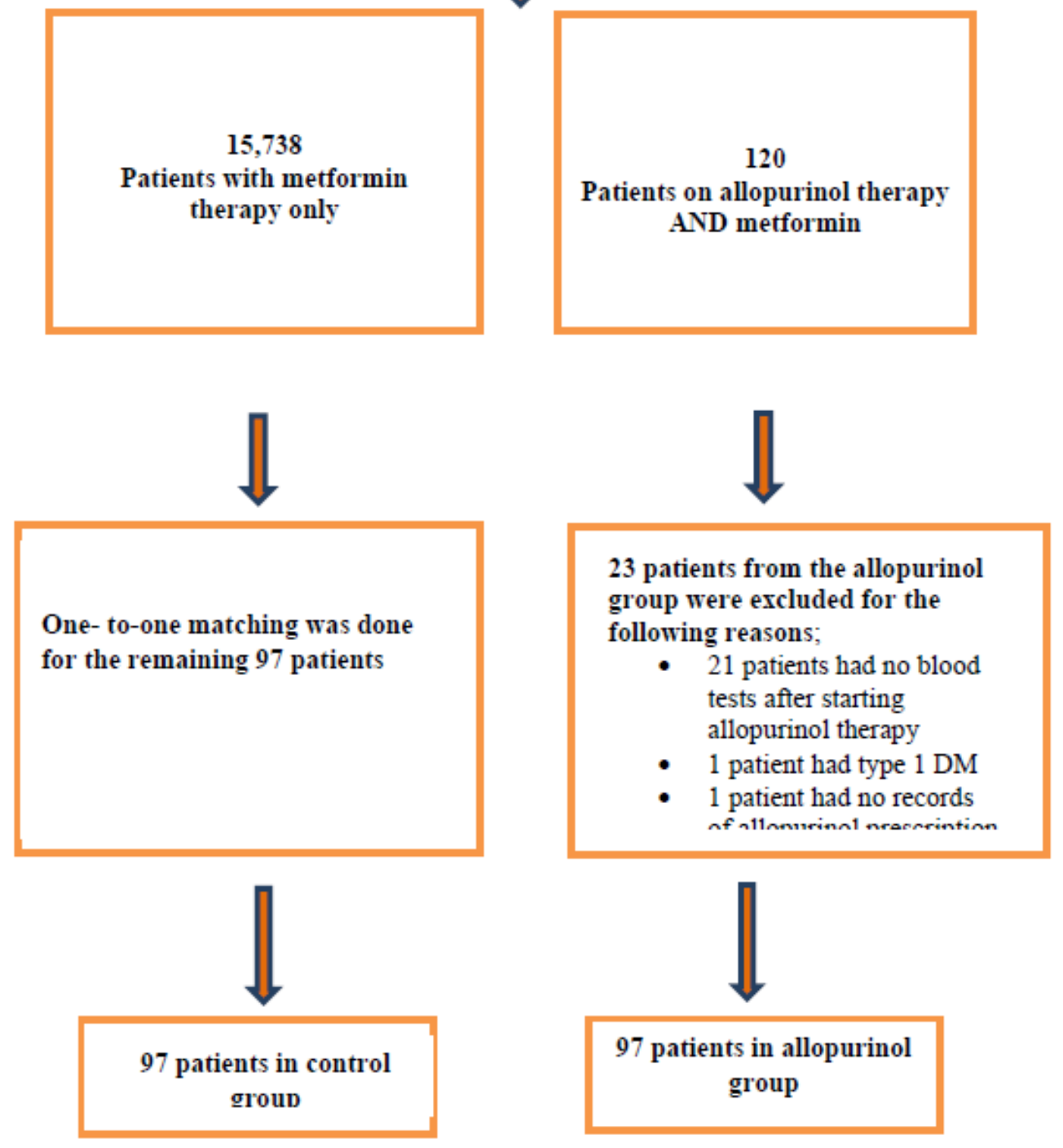

\section{Figure 1}

Study flow chart 


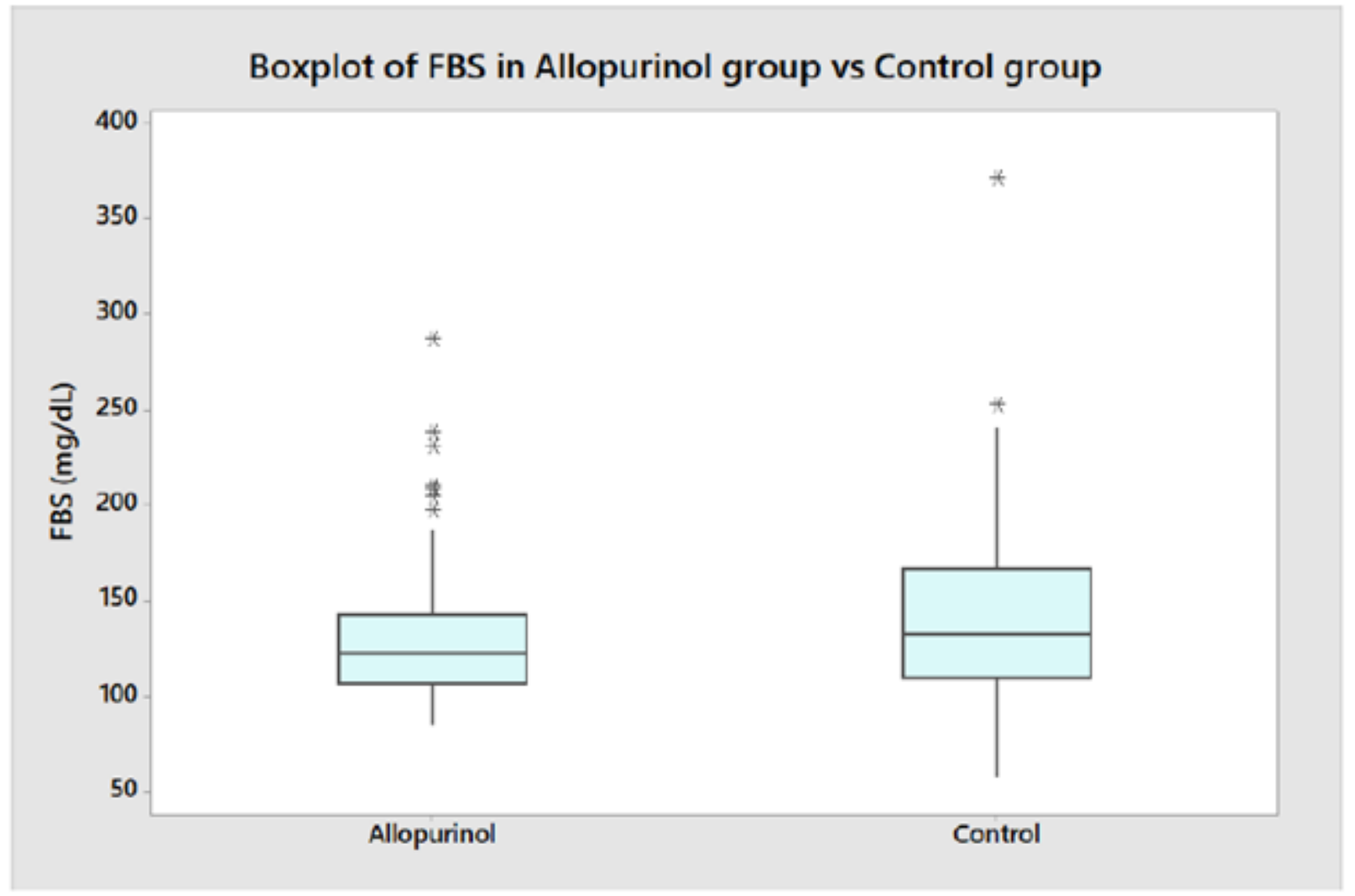

Figure 2

Comparison of FBS reading in both patients groups

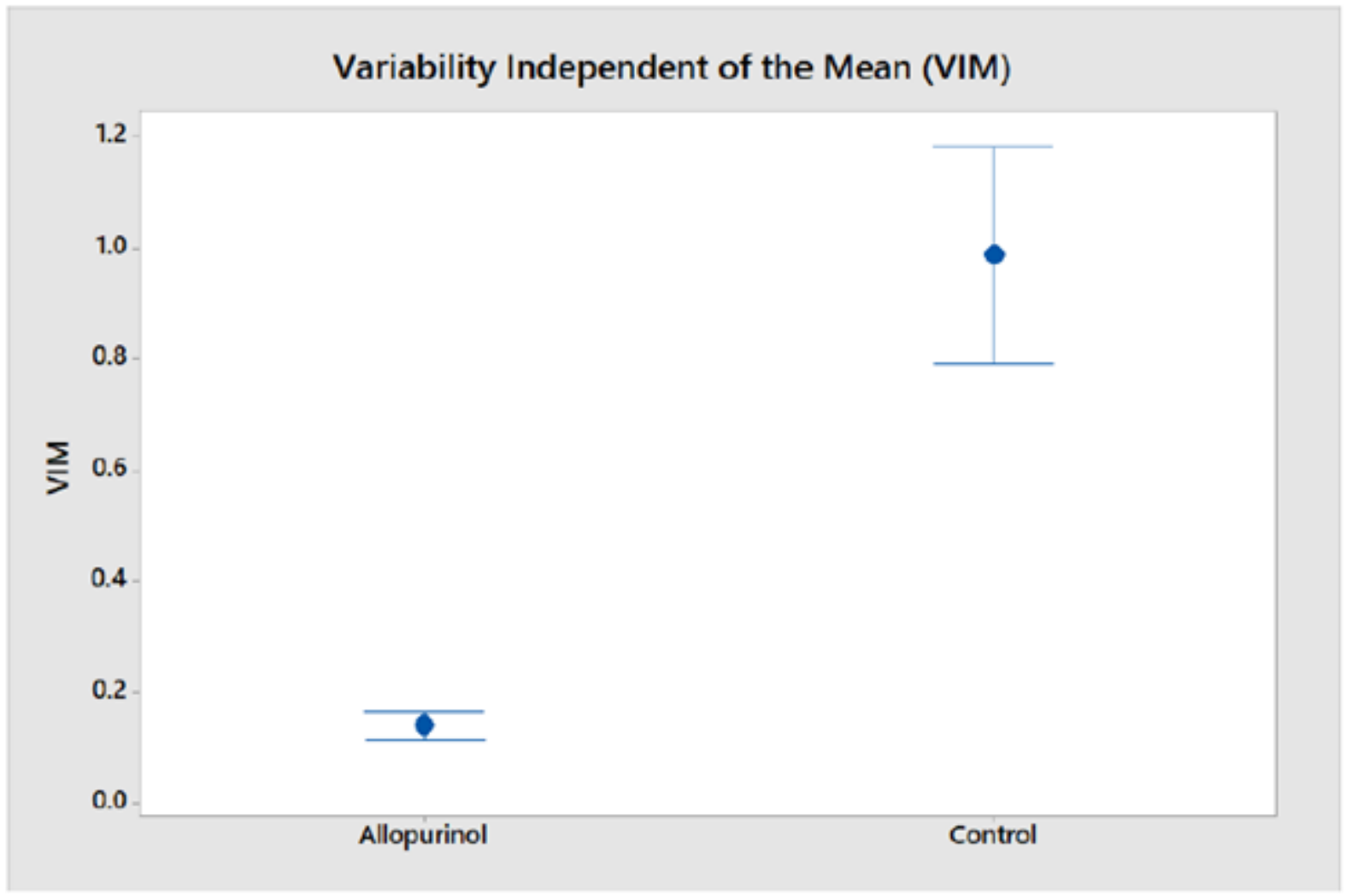


Figure 3

Comparison of VIM reading in both patients groups

\section{Supplementary Files}

This is a list of supplementary files associated with this preprint. Click to download.

- Table12v4.pdf 